\title{
GUÍA DE AUDITORÍA MÉDICA SOBRE DIAGNÓSTICO Y TRATAMIENTO DE LA ARTRITIS REUMATOIDEA.
}

\author{
MEDICAL AUDIT GUIDELINE ABOUT RHEUMATOID ARTHRITIS DIAGNOSIS AND TREATMENT.
}

Alejandra Babini ${ }^{1,3}$, Verónica Moreno ${ }^{1}$, Susana Bonardo ${ }^{2}$, Gisela Aguilera ${ }^{2}$, Maria Eugenia Farfan $^{2}$

\footnotetext{
1 Sociedad Argentina de Reumatología - Hospital Italiano de Córdoba.

2 Asociación de Auditoria y Calidad de Atención Médica de Córdoba (ASACAM).
}

3 Email de contacto: doctoraalejandrababini@outlook.com

\section{Conceptos clave}

A) Qué se sabe sobre el tema:

- La artritis reumatoidea es una patología inflamatoria frecuente, así como una causa destacada de incapacidad potencialmente tratable que se vincula con mayor riesgo de morbimortalidad general.

- Las opciones terapéuticas han mejorado de manera destacada la evolución de los pacientes.

B) Qué aporta este trabajo:

- Estas recomendaciones constituyen un recurso para orientar al médico auditor en la toma de decisiones, con la meta de optimizar no solo la calidad de la atención médica y de vida de los pacientes afectados, sino también los costos de las prestaciones.

\section{Resumen:}

Introducción: la artritis reumatoidea es una patología inflamatoria frecuente, así como una causa destacada de incapacidad potencialmente tratable que se vincula con mayor riesgo de morbimortalidad general. Las nuevas opciones terapéuticas han mejorado de manera destacada la evolución de los pacientes.

Objetivos: en este trabajo conjunto de la Asociación de Auditoria y Calidad de Atención Médica de Córdoba (ASACAM) y la Sociedad Argentina de Reumatología (SAR) se generaron recomendaciones para asistir especialmente a los médicos auditores en la toma de decisiones para mejorar la calidad de la atención médica y de vida de los pacientes y reducir los costos de las prestaciones en el enfoque de la artritis reumatoidea.

Destinatarios: además de médicos auditores, estas recomendaciones pueden expandirse a médicos generales, reumatólogos y clínicos, y eventualmente al público en general.

Conclusiones: se describen sugerencias para el diagnóstico y tratamiento de los pacientes con artritis reumatoidea, incluidas las terapias biológicas, sobe la base de las Guías de Práctica Clínica en el Tratamiento de la Artritis Reumatoidea (SAR, 2013), las resoluciones del Programa Médico Obligatorio y el denominado Sistema Único de Reintegro vigente en la Argentina.

Palabras clave: artritis reumatoide; terapia biológica; guía; auditoría médica; diagnóstico.

\section{Abstract:}

Introduction: rheumatoid arthritis is a frequent inflammatory disease and a leading cause of potentially-treatable disability. Rheumatoid arthritis is associated with an increased risk of general morbidity and mortality. New therapeutic options have dramatically improved the evolution of patients.

Objectives: in this joint work of the Association of Auditing and Quality of Medical Care of Córdoba (ASACAM) and the Argentine Society of Rheumatology (SAR), we generated recommendations to especially assist medical auditors in making decisions to improve the quality of the medical and life care of patients and reduce costs in the management of rheumatoid arthritis patients.

Intended population: in addition to medical auditors, these recommendations can be expanded to general practitioners, rheumatologists and clinicians, and eventually to the general public.

Conclusions: suggestions for the diagnosis and treatment of patients with rheumatoid arthritis (including biological therapies) are described, based on the Clinical Practice Guidelines for the Treatment of Rheumatoid Arthritis (SAR, 2013), the resolutions of the Compulsory Medical Program and the Unique Reimbursement System currently used in Argentina.

Keywords: arthritis, rheumatoid; biological therapy; guideline; medical audit; diagnosis. 


\section{Introducción}

La presente guía de auditoría médica sobre diagnóstico y/o tratamiento de la artritis reumatoidea (AR) incluye una serie de recomendaciones para los médicos auditores, que pueden ser extensivas para médicos generales, reumatólogos y clínicos, así como para el público en general.

Estas recomendaciones constituyen una herramienta para asistir especialmente al médico auditor en la toma de decisiones para mejorar la calidad de la atención médica y de vida de los afiliados y reducir los costos de las prestaciones.

Esta guía es el resultado de un trabajo conjunto de la Asociación de Auditoria y Calidad de Atención Médica de Córdoba (ASACAM) y la Sociedad Argentina de Reumatología (SAR) y se fundamenta en las Guías de Práctica Clínica en el Tratamiento de la Artritis Reumatoidea (SAR, 2013) 2, 3 , en las resoluciones del Programa Médico Obligatorio ${ }^{4}$ vigente en la Argentina, y sus modificaciones, (Resolución 1048/2014 de la Superintendencia de Salud) y el Sistema Único de Reintegro.

\section{Consideraciones iniciales}

La AR es una enfermedad crónica inflamatoria, progresiva, caracterizada por inflamación del tejido sinovial que conduce al deterioro ${ }^{5}$ funcional severo de las articulaciones involucradas, daño radiológico irreversible e incapacidad laboral. Puede comprometer otros órganos como pulmón, corazón, ojo, piel, vasculatura (vasculitis necrotizante), etc.

La AR es la patología inflamatoria más frecuente en Reumatología, se considera una causa frecuente de incapacidad (potencialmente tratable) ${ }^{6}$ y se asocia con incremento de la morbimortalidad, incluyendo un mayor riesgo de muerte. La AR causa daño irreversible, pero las opciones terapéuticas han mejorado ostensiblemente el devenir de los pacientes.

Diagnóstico y evaluación del paciente

La sospecha de la enfermedad, la pronta derivación al reumatólogo, el diagnóstico temprano y la iniciación de un tratamiento específico ${ }^{1}$ entre los 3 a 6 meses son fundamentales para poder revertir el curso natural de la enfermedad.

- La evaluación objetiva de pacientes con AR debe incluir ${ }^{1}$

- Recuento d articulaciones dolorosas y tumefactas.

- Evaluación del dolor y actividad de la enfermedad por escalas visuales análogas

- Valoración de la rigidez matinal.

- Determinación de la capacidad funcional por cuestionarios validados y culturalmente adaptados.

Se recomienda la evaluación del daño estructural con radiografías de manos, pies y articulaciones afectadas. Los códigos correspondientes para el PMO se resumen en la tabla 1. 


\begin{tabular}{|cc|}
\hline Tabla 1. Códigos de prácticas radiológicas para el PMO \\
\hline Área esquelética evaluada & Código PMO \\
\hline Antebrazo & 340213 \\
Cadera & 340211 \\
\hline Codo & 340213 \\
\hline Columna (raquis) & 340209 \\
\hline Fémur & 340211 \\
\hline Hombro & 340211 \\
\hline Húmero & 340211 \\
\hline Mano & 340213 \\
\hline Pelvis & 340211 \\
\hline Pie & 340213 \\
\hline Pierna & 340213 \\
\hline Rodilla & 340213 \\
\hline Tobillo & 340213 \\
\hline Tórax & 340301 \\
\hline
\end{tabular}

El laboratorio debe contemplar una rutina que incluya ${ }^{1}$

- Hemograma completo (recuento de glóbulos rojos, hematocrito, hemoglobina, recuento y fórmula leucocitaria, recuento de plaquetas), correspondiente al código 660475 del PMO

- Eritrosedimentación (VSG) como reactante de fase aguda, correspondiente al código 66029 del PMO

- Proteína C-reactiva (PCR), correspondiente al código 660761 del PMO. Este reactante de fase aguda demuestra correlación con la actividad de la enfermedad y con el daño radiológico a 5 años.

- Factor reumatoideo (FR), correspondiente al código 660598 del PMO. Si el FR basal es positivo, se aconseja su repetición al año ya que su persistencia en títulos elevados se asocia con mal pronóstico. Si al inicio de los síntomas el FR es negativo, se recomienda reiterar el estudio luego de los 6 a 12 meses del comienzo de la enfermedad. EI FR no es un anticuerpo específico de AR y sólo es positivo en el $35 \%$ de los casos de AR temprana.

- Anticuerpo antipéptido cíclico citrulinado (anti-CCP), correspondiente a los códigos 8284 del Nomenclador Bioquímico Único (NBU), con autorización de auditoría médica; código 778284 para PAMI y código 8284 para la Administración Provincial del Seguro de Salud (APROSS) y la Dirección de Asistencia Social del Personal Universitario (DASPU). La positividad del anti-CCP es predictiva de artritis persistente y de daño radiológico. Se recomienda solicitar anti-CCP en todo paciente con poliartritis indiferenciada que presente FR negativo. Niveles elevados de anti-CCP y FR se asocian con peores resultados clínicos a largo plazo y también, en forma independiente, con el desarrollo de manifestaciones extraarticulares. La repetición de la determinación de FR o antiCCP durante el primer año luego del inicio de la artritis no ofrecería información adicional, ya que se trata de un anticuerpo con valor predictivo para determinar pronóstico, no para seguimiento de actividad de enfermedad.

- Pruebas de función hepática: transaminasa glutámico oxalacética (TGO o AST), código 660874 del PMO; transaminasa glutámico pirúvica (TGP o ALT), código 660875 del PMO; fosfatasa alcalina, código 660357 del PMO; glutamil transpeptidasa, código 660420 del PMO.

- Pruebas de función renal: uremia, creatinina (código 660191 del PMO para creatinina en orina o sangre), orina completa (código 660711 para orina completa).

Estas determinaciones deben evaluarse con una periodicidad de 3 a 6 meses: nivel de evidencia $V$

Los factores de mal pronóstico se describen en la tabla 2. 


\begin{tabular}{l}
\hline Tabla 2. Factores de Mal Pronóstico en AR \\
\hline Presencia de factor reumatoideo \\
Anticuerpos antipéptidos cíclicos citrulinados \\
Actividad inflamatoria elevada \\
Grados elevados de discapacidad funcional \\
Retardo en la iniciación de un tratamiento específico \\
Presencia de manifestaciones extraarticulares \\
Bajo nivel socioeconómico \\
Presencia de erosiones óseas.
\end{tabular}

En el contexto de la evaluación del paciente con AR, la utilización de índices compuestos de actividad de la enfermedad permite un mejor control de la misma. Los índices más utilizados son Disease Activity Score (DAS28), Simplified Disease Activity Index (SDAI) y Clinical Disease Activity Index (CDAI).

El índice DAS28 es un instrumento simplificado y validado ${ }^{7}$, que tiene en cuenta solamente 28 articulaciones concretas para realizar el cálculo del puntaje. Se cuantifica el número de articulaciones tumefactas y dolorosas entre las 28 evaluadas, así como la VSG y la evaluación global del estado de salud por parte del paciente, por medio de una escala analógica visual. El DAS28 permite evaluar y discriminar entre alta y baja actividad de la enfermedad (nivel de evidencia I de acuerdo con las guías SAR 2013) ${ }^{1}$. Además, presenta habilidad para detectar recaídas de la actividad de la enfermedad (nivel de evidencia II). 1 Los cambios en el DAS28 de 1.2 puntos se consideran significativo y se recomienda efectuar su cálculo cada 3 meses. ${ }^{1}$ Si bien el puntaje DAS28 no sustituye a la evaluaciónclínica cuidadosa de los pacientes, se lo considera una guía útil para las decisiones terapéuticas ${ }^{8}$.

EI DAS28-PCR es una variante de DAS28 que no utiliza la velocidad de sedimentación globular, sino el recuento de la PCR. La interpretación y la distribución de articulaciones para el cálculo del puntaje DAS28 se describen en la figura 1. 


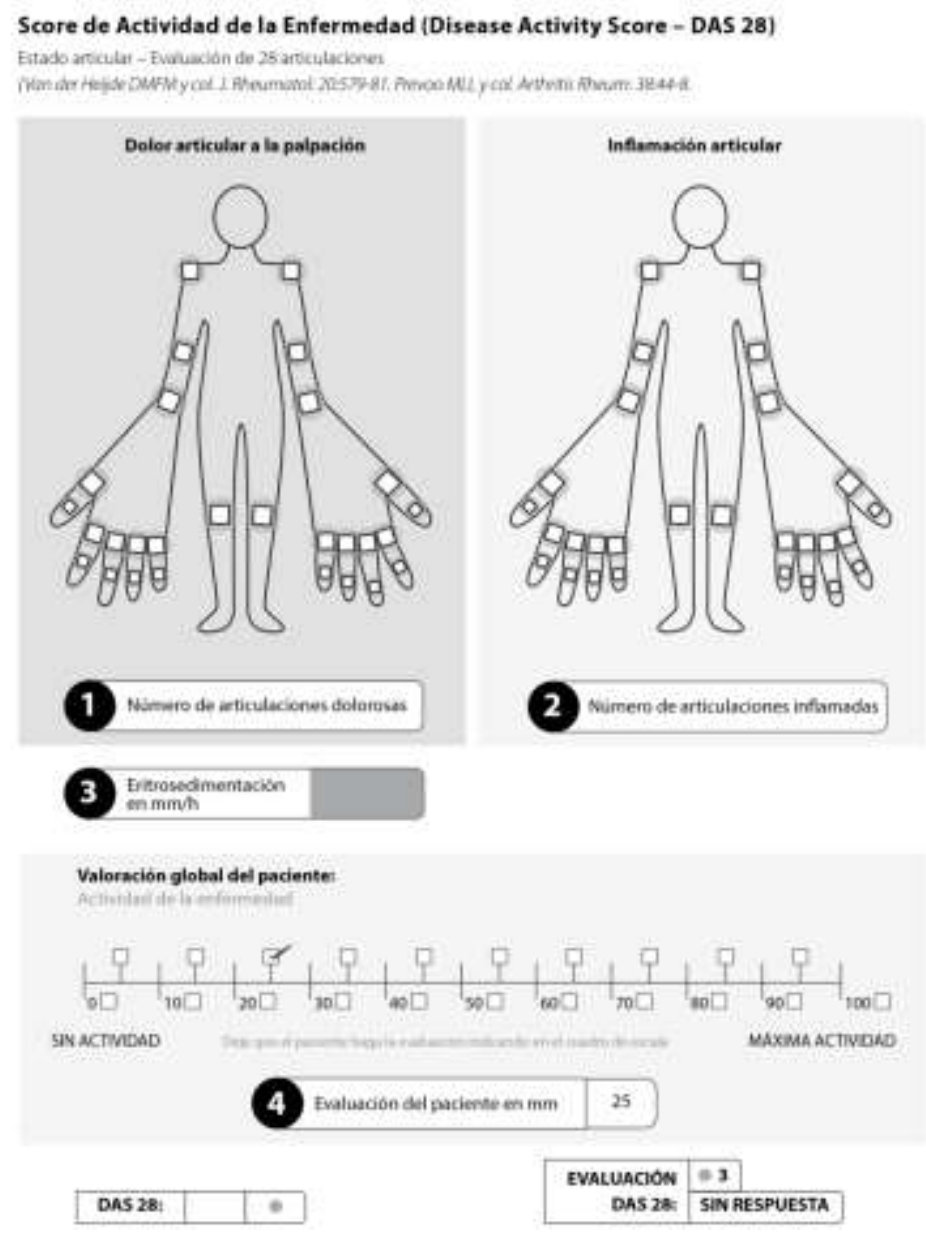

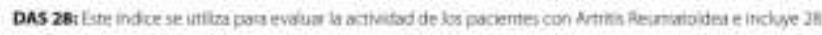

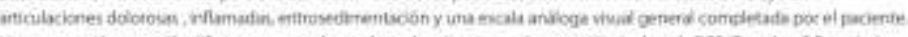

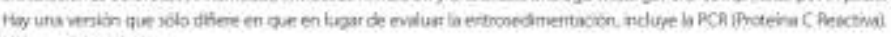
Elangovade 020.4

So consiban tua axtidatad $<433$

Modieada acrivilua $>3225,1$

Ahactivided $>5,1$

Figura 1. Puntaje DAS28

El puntaje $\mathrm{SDAl}^{9}$ se calcula por medio de la sumatoria de:

- $\quad$ número de articulaciones dolorosas (recuento de 28)

- $\quad$ número de articulaciones tumefactas (recuento de 28)

- $\quad$ evaluación global de la enfermedad por evaluación realizada por el paciente (escala de 0 a $10 \mathrm{~cm}$ )

- $\quad$ evaluación global de la enfermedad por evaluación realizada por el médico (escala de 0 a $0 \mathrm{~cm}$ )

- $\quad$ PCR (mg/dl)

El puntaje CDAI8 surge de la sumatoria de las 4 primeras variables del SDAI, pero no toma en cuenta el valor de la PCR. En la tabla 3 se sintetizan los conceptos de remisión y nivel de actividad para las escalas DAS28, SDAI, CDAl y Routine Assessment of Patient Index Data 3 (RAPID3) ${ }^{10}$, mientras que los estadios de la enfermedad se describen en la figura 2.

Tabla 3. Escalas de validación de actividad de la AR

\begin{tabular}{|l|c|c|c|c|}
\hline \multicolumn{1}{|c|}{ Escala } & Remisión & Baja actividad & $\begin{array}{c}\text { Moderada } \\
\text { actividad }\end{array}$ & Alta actividad \\
\hline DAS 28 & $>2.6$ & $\leq 3.2$ & $\leq 5.1$ & $>5.1$ \\
\hline SDI & $\leq 3.3$ & $\leq 11$ & $\leq 26$ & $>26$ \\
\hline CDAI & $\leq 2.8$ & $\leq 10$ & $\leq 22$ & $>22$ \\
\hline RAPID3 & $\leq 1.0$ & $\leq 2.0$ & $<4.0$ & $>4.0$ \\
\hline
\end{tabular}




\title{
Los estadíos de AR
}

\author{
Figura 2. Estadíos de AR \\ fadaptada de Smolen is et of EULAP recommendations \\ for the management of rheumatoid arthritis with synthetic and biological \\ disease-modifying antirheumatic drugs: 2016 update
}

Tratamiento de la AR

En la actualidad se recomienda el modelo de tratamiento por objetivos (treat to target, T2T). El T2T se focaliza en establecer blancos terapéuticos de respuesta clínica, compartir las decisiones con el paciente, efectuar control estricto de la actividad de la enfermedad y realizar ajustes del tratamiento cuando esos objetivos no se logran ${ }^{11,12}$.

El objetivo primario del tratamiento de la AR debiera ser el estado de remisión clínica, definida (bajo medicación) como la ausencia de signos y síntomas de enfermedad inflamatoria activa ${ }^{1}$. Hasta lograr el objetivo terapéutico deseado, el tratamiento debiera ser ajustado por lo menos cada 3 meses ${ }^{1}$.

Se deben obtener y registrar medidas de actividad de la enfermedad regularmente. En pacientes con actividad de la enfermedad de moderada a grave se deben realizar cada 1 a 3 meses y en pacientes con bajo nivel de actividad o remisión, cada 3 meses.

El tratamiento por objetivo deseado debe mantenerse durante todo el curso de la enfermedad ${ }^{1}$.

La droga modificadora de la AR (DMAR) dependerá de la forma clínica de comienzo (leve, moderado o severo), de las condiciones del paciente y de las enfermedades concomitantes. Habitualmente la droga de elección sigue siendo metotrexate (DMAR convencional), en una dosis que varía entre 7,5 mg y 25 $\mathrm{mg} / \mathrm{semanales}$. Se considera falla al tratamiento con metotrexate cuando este fármaco se indica entre 3 a 6 meses en dosis máximas (20 a $25 \mathrm{mg}$, habiendo utilizado la vía intramuscular o subcutánea) sin alcanzar la baja actividad de enfermedad o remisión. 
Las drogas biológicas deben ser recomendadas en pacientes con AR activa que no han respondido al tratamiento combinado con al menos dos DMAR convencionales y/o al metotrexate en dosis máxima de $25 \mathrm{mg} / \mathrm{semanales,} \mathrm{o} \mathrm{que} \mathrm{han} \mathrm{presentado} \mathrm{intolerancia} \mathrm{o} \mathrm{la} \mathrm{presencia} \mathrm{de} \mathrm{eventos} \mathrm{adversos} \mathrm{a} \mathrm{las} \mathrm{mismas,}$ después de 3 a 6 meses como máximo u 8 a 12 semanas según lo propuesto por Grupo Latinoamericano de Estudio de Artritis Reumatoide ${ }^{3}$ (GLADAR) y el Consenso de la Pan-American League of Associations for Rheumatology (PANLAR).

Los biológicos DMAR pueden ser agregados o bien reemplazar a los DMAR convencionales en el tratamiento. Deben ser considerados drogas de primera elección sólo en aquellos pacientes con AR activa y contraindicaciones formales a los DMAR convencionales ${ }^{1}$.

Los agentes biológicos producen mejoría significativa de los síntomas y signos clínicos y de laboratorio; además, existe evidencia de que retardan la progresión radiológica de la enfermedad ${ }^{1}$.

Antagonistas del TNF

Los agentes biológicos antagonistas del factor de necrosis tumoral (TNF) alfa son recomendados para el tratamiento de pacientes con AR activa que no han respondido en un tiempo adecuado a la monoterapia con metotrexate a dosis máximas de hasta $25 \mathrm{mg} / \mathrm{semana}$, o a metotrexate combinado con otra DMAR convencional, o que han presentado intolerancia a las mismas. Se destaca que no es necesario que un paciente con AR activa reciba diferentes cursos de otras DMAR (sulfasalazina, hidroxicloroquina, leflunomida, etc.) antes de iniciar un agente anti-TNF.

La indicación no debe estar limitada solamente al paciente que tiene un puntaje DAS28 $\geq 5,1$ debido a que existen individuos con actividad persistente de la enfermedad que no alcanzan dicho valor. Los anti-TNF disponibles y su posología se resumen en la tabla 4.

\begin{tabular}{|c|c|c|c|}
\hline Fármaco & Dosis & Intervalo & $\begin{array}{c}\text { Vía de } \\
\text { administración }\end{array}$ \\
\hline Adalimumab & $40 \mathrm{mg}$ & Cada 2 semanas & Subcutánea \\
\hline Infliximab & 3 a $10 \mathrm{mg} / \mathrm{kg}$ & $\begin{array}{c}\text { Semanas } 0,2 \text { y } 6 \text {; luego cada } 8 \\
\text { semanas }\end{array}$ & Intravenosa lenta \\
\hline Etanercept & $50 \mathrm{mg}$ & Semanal & Subcutánea \\
\hline $\begin{array}{c}\text { Certolizumab } \\
\text { pegol }\end{array}$ & $\begin{array}{l}400 \mathrm{mg} \text { (inicio), } 200 \mathrm{mg} \\
\text { (mantenimiento) }\end{array}$ & $\begin{array}{c}\text { Cada } 2 \text { semanas (o } 400 \mathrm{mg} \text { cada } 4 \\
\text { semanas) }\end{array}$ & Subcutánea \\
\hline Golimumab & $50 \mathrm{mg}$ & Cada 4 semanas & Subcutánea \\
\hline
\end{tabular}

Otros biológicos DMAR ${ }^{1}$

Abatacept es un modulador de la señal de coestimulación requerida para la activación completa de los linfocitos T. Se indica en dosis de $10 \mathrm{mg} / \mathrm{kg}$ en infusión intravenosa durante 30 minutos, en los días 1, 15, 29, para administrarse luego mensualmente. No requiere medicación previa. También se encuentra disponible una formulación de $125 \mu \mathrm{g}$ para administración subcutánea semanal.

Rituximab es un anticuerpo monoclonal quimérico anti- CD20, aprobado por FDA y en Europa para el tratamiento de pacientes con AR con inadecuada respuesta a $>1$ anti-TNF. Se indican $1000 \mathrm{mg}$ por vía intravenosa en los días 1 y 15, con premedicación (100 mg de metilprednisolona intravenosa).

Tocilizumab es un anticuerpo monoclonal humanizado recombinante, dirigido contra el receptor humano de la interleuquina 6. Está indicado en combinación con metotrexate para el tratamiento de la AR activa de moderada a grave en pacientes adultos con respuesta inadecuada o intolerancia a un tratamiento previo con uno o más DMAR o con anti-TNF. En estos pacientes, tocilizumab puede ser administrado como monoterapia en caso de intolerancia o tratamiento inadecuado con metotrexate. 
La dosis recomendada es de $8 \mathrm{mg} / \mathrm{kg}$ por infusión intravenosa, a pasar en una hora, con una dosis mínima de $480 \mathrm{mg}$ y una dosis máxima de $800 \mathrm{mg}$, con intervalos de 4 semanas. Se dispone además de una formulación de $162 \mathrm{mg}$ para aplicación subcutánea semanal.

\section{DMAR sintéticos}

Tofacitinib es un inhibidor selectivo de las quinasas Janus (JAK) 1 y 3, mediante el bloqueo competitivo en el sitio de unión del ATP. Está indicado para el tratamiento de pacientes con AR moderada a severa, que no hayan respondido o tengan contraindicación para metotrexate (solo o en combinación con otros DMAR convencionales) u otros DMAR sintéticos o agentes biológicos. Tofacitinib puede utilizarse combinado con metotrexate $u$ otros DMAR convencionales, o bien en monoterapia. No debe administrarse conjuntamente con agentes biológicos, ni otros inmunosupresores potentes como azatioprina o ciclosporina. La dosis aprobada es de $5 \mathrm{mg}$ dos veces al día por vía oral y se dispone de una formulación para administración de $11 \mathrm{mg}$ en una única toma diaria ${ }^{14}$.

Baricitinib es un inhibidor selectivo de las JAK 1 y 2, indicado en dosis de $4 \mathrm{mg}$, una vez al día, con la posibilidad de administrarse en dosis de $2 \mathrm{mg} / \mathrm{día}$ en pacientes mayores de 75 años o con insuficiencia renal; además, puede ser apropiado para pacientes con antecedentes de infecciones crónicas o recurrentes. No se encuentra disponible aún la aprobación regulatoria por la Administración Nacional de Medicamentos, Alimentos y Tecnología Médica (ANMAT).

\section{Medicamentos biosimilares}

Los medicamentos biológicos similares, productos bioterapéuticos similares o bien biocomparables han sido definidos por la Organización Mundial de la Salud y la Agencia Europea de Medicamentos como "un producto bioterapéutico similar en términos de calidad, seguridad y eficacia a un producto bioterapéutico de referencia ya comercializado" ${ }^{15}$.

En términos prácticos, un biosimilar es un agente biológico que ha demostrado ser similar o comparable a la medicación original en términos de eficacia ${ }^{16}$, seguridad y calidad del producto, habiendo sido esto demostrado por ejercicios comparativos rigurosos y luego aprobado por la ANMAT.

Se define como intercambiabilidad a aquella situación en la que dos productos bioequivalentes (es decir, idénticos desde el punto de vista farmacéutico) pueden ser intercambiados, uno por el otro, sin un riesgo significativo de un resultado adverso para la salud. El principal dilema que se plantea con los biosimilares es que, por definición, son diferentes (pero comparables) a la droga de referencia original, siendo por lo tanto no intercambiables ${ }^{15}$. La definición de intercambiabilidad es compleja, debe ser formulada por las agencias regulatorias e incluir la totalidad de la evidencia.

El concepto "sustitución automática" hace referencia a cuando la sustitución sucede al momento de la dispensación, cuando el farmacéutico (en la farmacia o en el hospital) elige cambiar un producto sin el consentimiento previo del médico tratante y mucho menos del paciente ${ }^{15}$.

La SAR desaconseja la implementación de sustitución automática para este tipo de drogas sin informar al médico tratante, debido a su complejo mecanismo de acción y perfil de seguridad (especialmente inmunogenicidad $)^{1}$. El cambio de una droga de referencia por un biosimilar debe ser evaluado idealmente en el contexto clínico de cada paciente, lo cual solo puede ser realizado por el medico reumatólogo tratante. ${ }^{1}$

A modo de conclusión, se señala que el Sistema Único de Reintegro vigente en nuestro país, destaca en las resoluciones 1200/2012 y 046/2017 de la Superintendencia de Servicios de Salud que, en enfermedad osteoarticular, se solicita la siguiente documentación para proceder al reintegro económico:

- Historia clínica.

- Estudios efectuados para llegar al diagnóstico de certeza.

- Laboratorio inmunológico.

- Tratamientos previos efectuados detallando drogas utilizadas, tiempo, dosis y asociaciones medicamentosas. 
- $\quad$ Para la solicitud de cualquiera de los agentes biológicos (salvo que no se trate de aquellos correspondientes a medicación de primera línea), se deberá demostrar el fracaso en la respuesta a terapias previas con otros fármacos.

- Deberá presentarse la fundamentación médica del uso del medicamento sobre el cual se solicita el reintegro con detalle de dosis utilizada, tiempo y respuesta, firmada y sellada por el especialista y el auditor médico del Agente de Seguro de Salud.

- Consentimiento informado.

Limitaciones de responsabilidad: No hay conflicto de intereses. La investigación fue aprobada por los comités de ética de las instituciones participantes

Fuentes de apoyo: Abbvie S.A. (colaboración financiera).

\section{Bibliografía}

1. Grupo de Estudio de Artritis Reumatoidea. Actualización de las Guías de Práctica Clínica en el Tratamiento de la Artritis Reumatoidea [Internet]. Buenos Aires: Sociedad Argentina de Reumatología; 2013 [cited 2018 Aug 12]. Available from: http://www.reumatologia.org.ar/docs/guias_sar_2013.pdf.

2. Programa Médico Obligatorio de Emergencia. Resolución 201/2002 [Internet]. Buenos Aires: Ministerio de Salud de la Nación; 2002 [cited 2018 Aug 10]. Available from: https://www.sssalud.gob.ar/pmo/res s 02 201.pd

3. Ministerio de Salud de la Nación. Resolución 310/2004 [Internet]. Buenos Aires; 2004 [cited 2018 Aug 11]. Available from: https://www.sssalud.gob.ar/normativas/consulta/000595.pdf.

4. Superintendencia de Servicios de Salud. Resolución 1048/14 [Internet]. Buenos Aires: Ministerio de Salud de la Nación; 2014 [cited 2018 Aug 11]. Available from: https://bit.Iy/2RUmv8X

5. Choy EH, Panayi GS. Cytokine pathways and joint inflammation in rheumatoid arthritis. N Engl J Med. 2001 Mar 22;344(12):907-16.

6. van den Hoek J, Boshuizen HC, Roorda LD, Tijhuis GJ, Nurmohamed MT, van den Bos GA, et al. Mortality in patients with rheumatoid arthritis: a 15-year prospective cohort study. Rheumatol Int. 2017 Apr;37(4):487-493.

7. Prevoo MLL, van't Hof MA, Kuper HH, van Leeuwen MA, van de Putte LBA, van Riel PLCM. Modified Disease Activity Scores that include Twenty-Eight-Joint Counts. Arthritis Rheum. 1995 Jan;38(1):44-8.

8. Fransen J, Stucki G, van Riel PLCM. Rheumatoid arthritis measures: Disease Activity Score (DAS), Disease Activity Score-28 (DAS28), Rapid Assessment of Disease Activity in Rheumatology (RADAR), and Rheumatoid Arthritis Disease Activity Index (RADAl). Arthritis \& Rheumatism 2003 Oct 15;49(5S):S214-S224.

9. Aletaha D, Smolen J. The Simplified Disease Activity Index (SDAI) and the Clinical Disease Activity Index (CDAl): a review of their usefulness and validity in rheumatoid arthritis. Clin Exp Rheumatol. 2005 Sep-Oct;23(5 Suppl 39):S100-8.

10. Pincus T, Yazici Y, Bergman MJ. RAPID3, an index to assess and monitor patients with rheumatoid arthritis, without formal joint counts: similar results to DAS28 and CDAl in clinical trials and clinical care. Rheum Dis Clin North Am. 2009 Nov;35(4):773-8, viii.

11. Smolen JS, Aletaha D, Bijlsma JW, Breedveld FC, Boumpas D, Burmester G, Combe B, Cutolo M, de Wit M, Dougados M, Emery P, Gibofsky A, Gomez-Reino JJ, Haraoui B, Kalden J, Keystone EC, Kvien TK, McInnes I, Martin-Mola E. Montecucco C, Schoels M, van der Heijde D; T2T Expert Committee. Treating rheumatoid arthritis to target: recommendations of an international task force. Ann Rheum Dis. 2010 Apr;69(4):631-7.

12. Smolen JS, Landewé R, Breedveld FC, Buch M, Burmester G, Dougados M, et al. EULAR recommendations for the management of rheumatoid arthritis with synthetic and biological disease-modifying antirheumatic drugs: 2013 update. Ann Rheum Dis. 2014 Mar;73(3):492-509.

13. Cardiel MH; Latin American Rheumatology Associations of the Pan-American League of Associations for Rheumatology (PANLAR); Grupo Latinoamericano de Estudio de Artritis Reumatoide (GLADAR). First Latin American position paper on the pharmacological treatment of rheumatoid arthritis. Rheumatology (Oxford). 2006 Jun;45 Suppl 2:ii7-ii22.

14. Administración Nacional de Medicamentos, Alimentos y Tecnología Médica (ANMAT). Disposición 2776/2013 [Internet]. Buenos Aires; 2013 [cited 2018 Aug 13]. Available from https://bit.ly/2qXHZ9H

15. World Health Organization Expert Committee on Biological Standardization. Annex 2. Guidelines on evaluation of similar biotherapeutic products (SBPs). Geneva: World Health Organization; 2013. WHO Technical Report Series no. 977.

16. Alerany C, Armellini A, Bosó V, Calvo G, Cruz E, Diego L, et al. Libro blanco de los medicamentos biosimilares en España: calidad sostenible. La garantía del acceso universal a medicamentos clave. Disponible en: http://www.fgcasal.org/publicaciones/Libro_Blanco_de_los_Medicamentos_Biosimilares.pdf (consultado el 12 de agosto de 2018). 